\title{
Engineering the electron g-factor anisotropy in double quantum wells
}

\author{
J.E. Leon Padilla ${ }^{1}$, M. A. Toloza Sandoval2 ${ }^{*}$, E. A. de Andrada e Silva ${ }^{3}$, A. Ferreira da Silva ${ }^{1}$, G. C. La Rocca \\ 1 Instituto de Física, Universidade Federal da Bahia, Salvador, Brazil. \\ 2 Instituto de Física de São Carlos, Universidade de São Paulo, Brazil. \\ ${ }^{3}$ Instituto Nacional de Pesquisas Espaciais, São José dos Campos, Brazil. \\ ${ }^{4}$ Scuola Normale Superiore di Pisa, Italy.
}

*tolozasandoval@gmail.com

Keywords: effective g factor, quantum tunneling, spin-orbit interaction.

We investigate the quantum tunneling effects on the electron $g$ factor and its main anisotropy in semiconductor double quantum wells (DQWs). With respect to single QWs, these structures introduce a new degree of freedom, given by the tunnel coupling parameter, and can be very helpful in the g-factor engineering. The eight-band effective-mass Hamiltonian for electrons in III-V double QWs and in the presence of an external magnetic field (applied both in the QW plane and along the growth direction) is solved for the $\mathrm{g}$ factor within first-order perturbation theory. We then calculate the g-factor anisotropy as a function of the QW width and the inter-well barrier length in typical AIGaAs/GaAs DQWs. The obtained results reproduce exactly the well-known single QW results in the corresponding limits, i.e. $L_{b}=0$ and very large, and explicitly show how the well-width dependence of the g-factor anisotropy changes with Lb, interpolating between these two limiting single $\mathrm{QWs}$, with well width $2 \mathrm{~L}_{w}$ and $\mathrm{L}_{w}$ respectively.

\section{Introduction}

The effective $\mathrm{g}$ factor for electrons confined in semiconductor nanostructures can be strongly renormalized by the spin-orbit interaction and manipulated through the engineering of the confining potential [1-5]. There is today a clear and increasing technological interest in these effects in view of the development of both, new spintronic devices and quantum computation schemes, involving in particular the Majorana bound states in coupled nanowires. However, the determination and control of the electron $\mathrm{g}$ factor in general nanostructures is far from simple and mostly an open problem yet. For single GaAs quantum wells (QWs), it is by now clear, both theoretical [1-3] and experimentally [4,5], that the electron effective $\mathrm{g}$ factor interpolates from the bulk value in the AIGaAs barrier to the GaAs bulk value, as the well width goes from zero to infinity. More interestingly is that due to the break of translation symmetry along the growth direction, the effective $\mathrm{g}$ factor for electrons in QWs develops an anisotropy $\Delta \mathrm{gQW}^{\mathrm{QW}}$, corresponding to the difference between the $\mathrm{g}$ factor for magnetic fields applied in the QW plane and that for fields aligned along the growth direction. Such anisotropy has been clearly observed experimentally [4,5] but the agreement with the available theories [1-3] is still not complete; there are, in particular, discrepancies between the theories regarding the anisotropy dependence with the well width $[1,2]$. In order to shine some light to this problem and also to understand the possible implications of the tunnel coupling in the more general double well structures (DQWs) illustrated in Figure 1, we calculate the effective $g$ factor for electrons in these DQWs and investigate its anisotropy as a function of the well width $L_{w}$ and the inter-well barrier width $L_{b}$, i.e. $\Delta g^{D Q W}\left(L_{w} ; L_{b}\right)$.

(a)

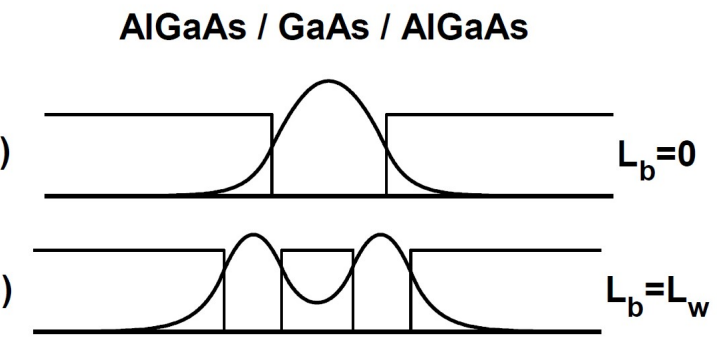

(c)

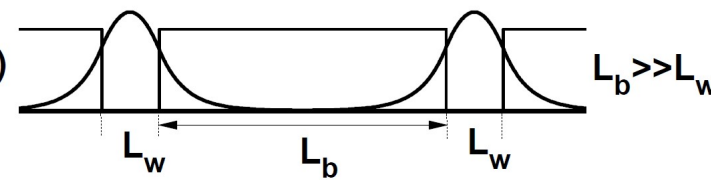

Figure 1. Scheme of GaAs DQW structures, with the conduction band profile, the actual ground state wave functions for confined electrons and well width $\mathrm{L}_{\mathrm{w}}$ and inter-well barrier width $\mathrm{L}_{\mathrm{b}}$. 


\section{Methods and Results}

We consider DQWs consisted of two GaAs/AIGaAs QWs with the same well width $L_{w}$ coupled by an inter-well AIGaAs barrier of width $L_{b}$, which represents the tunnel coupling parameter. In both extremes, $\mathrm{L}_{b}=0$ and very large, the $\mathrm{DQW}$ structure is reduced to single QWs. Here with these DQW calculations we can further test the above mentioned single $\mathrm{QW}$ results and also investigate how the anisotropy of DQW g-factor interpolates between these two single QW limits. We follow the same method of calculation used in References 2 and 3, which from the eight-band Kane model for the bulk, derives an effective-mass Hamiltonian for the conduction-band envelope function, considering both the band-offsets and the applied magnetic-field, and then solves it for the $\mathrm{g}$ factor, within first-order perturbation theory $[2,3]$.

Here, instead of solving for the single QW band profile [1-3], we solve it for the DQW band profile. The details of the method, with the discussion of the approximations made, can be found in Ref. [3]; and the DQW unperturbed solution used is as given in Ref. [6]. The $\mathrm{g}$ factor is calculated for fields applied in the QW plane and perpendicular to it (i.e. along the growth direction) and the difference gives the g-factor anisotropy; in Fig. 2, for example, we show the results for g-factor anisotropy in AIGaAs/GaAs DQWs as a function of the well width $L_{w}$, and for different values of the coupling parameter (or inter-well barrier width).

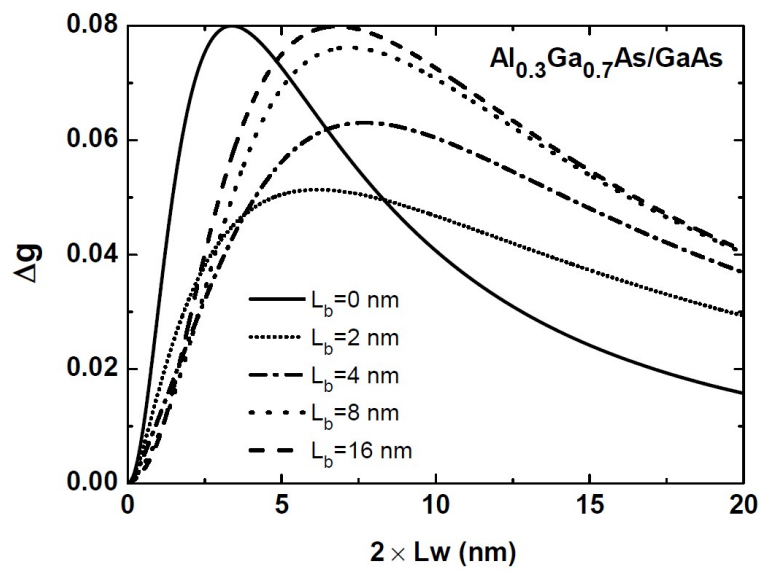

Figure 2. Obtained results for the effective g-factor anisotropy for electrons confined in AIGaAs/GaAs double quantum wells as a function of the well width $\left(L_{w}\right)$ and for varying coupling strength, i.e. $L_{b}$.

\section{Discussion}

Firstly, it is interesting to note that $\Delta \mathrm{g}$ is positive, which means that the in-plane $\mathrm{g}$ factor is larger than that along the growth direction. As expected, in the two single QW limits mentioned above, i.e. for $L_{b}=0$ and very large, the present DQW results reduce exactly to the known QW results, with the g-factor anisotropy going to zero both for very small and very large $L_{w}$. Figure 2 also shows how the function $\Delta g\left(L_{w}\right)$ is modified due to the tunnel coupling between the two wells. One can see that, starting from zero, as soon as the interwell barrier width is not negligible there is a large decrease in the maximum anisotropy and in the anisotropy variation with $\mathrm{L}_{w}$. If one continues to increase $L_{b}$, the $\Delta g^{D Q W}\left(L_{w}\right)$ curve then slowly returns to the known single $\mathrm{QW}$ case, i.e. $\Delta g^{D Q W}\left(L_{w} ; L_{b}>>L_{w}\right)=\Delta g^{Q W}\left(L_{w}\right)$, while $\Delta g^{D Q W}\left(L_{w} ; 0\right)=$ $\Delta \mathrm{g}^{\mathrm{QW}}\left(2 \mathrm{~L}_{\mathrm{w}}\right)$. Note that the absolute value of the $\mathrm{g}$ factor anisotropy is however still limited by the single QW maximum anisotropy.

Summarizing, we have investigated the effective $g$ factor for electrons in semiconductor DQWs and presented specific results for the gfactor anisotropy in AIGaAs/GaAs DQWs. New and different variations for the g-factor anisotropy as a function of both barrier and well width are obtained which describe effects of the quantum tunneling in the electron g-factor engineering, which in turn is fundamental to the development of spintronic applications.

\section{Acknowledgments}

The authors thank the Brazilian agencies CNPq, FAPESP, CAPES and FAPESB for financial support.

\section{References}

[1] Ivchenko, E. L. and Kiselev, A. A. Sov. Phys. Semicond. 1992, 26 827.

[2] Toloza Sandoval, M.A.; Silva, A. F.; Andrada e Silva, E. and La Rocca, G. C. Phys. Rev. B. 2012, 86, 195302.

[3] Toloza Sandoval, M.A.; Silva, A. F.; Andrada e Silva, E. and La Rocca, G. C. Semicond. Sci.Technol. 2016, 31, 115008.

[4] Le Jeune, P.; Robart, D.; Marie, X.; Amand, T.; Brosseau, M.; Barrau, J. and Kalevcih, V. Semicond. Sci.Technol. 1997, 12, 380.

[5] Malinowski, A. and Harley, R. T. Phys. Rev. B. 2000, 62, 2051.

[6] Bastard, G., "Wave mechanics applied to semiconductor heterostructure”, Monographies de Physique, Les Editions de Physique, Les Ulis Cedex 